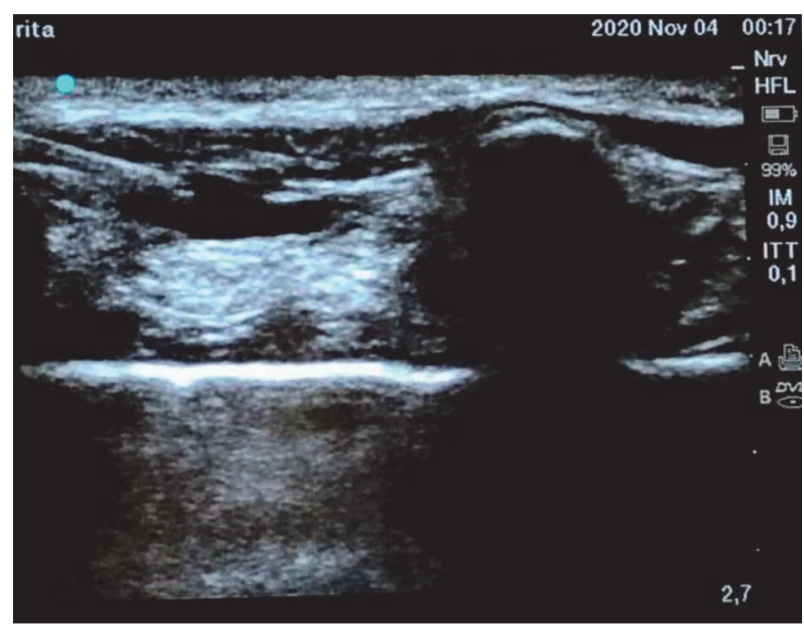

Abstract 206 Figure 2

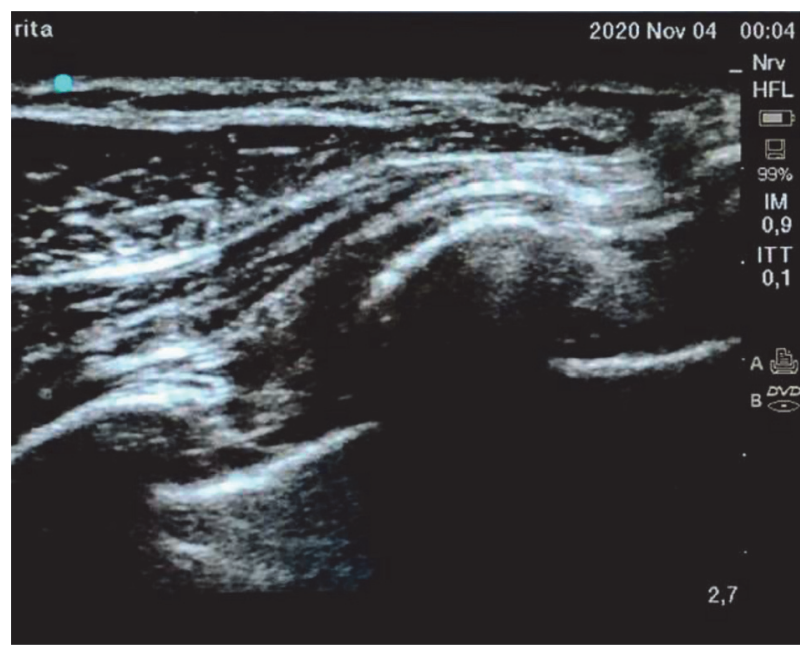

Abstract 206 Figure 3

anesthetics required during surgery. No pain (movement/rest). No morphine required. No peri-operative complications recorded.

Conclusions We performed US-guided inter-fascial plane blocks with high efficacy and safety. Our patient was successfully surgically treated. Regional anesthesia allowed early recovery, reduced postoperative opioid/non-opioid analgesics consumption together with early home discharge and cost reduction.

\section{IMPLEMENTATION OF TRAINING IN ULTRASOUND GUIDED REGIONAL ANAESTHETIC 'PLAN A' NERVE BLOCKS DURING THE COVID PANDEMIC - TRAINING MUST GO ON!}

F Moosa*, N Sadavarte, N Pinnamaneni, D Luff, A Hassan, N Bedforth. Nottingham University Hospitals NHS Trust, Nottingham, UK

\subsection{6/rapm-2021-ESRA.207}

Background and Aims There has been a significant impact on anaesthetic teaching and training within the UK owing to the COVID-19 pandemic. Most of the teaching needs are met using virtual methods. Hands on skills such as regional anaesthesia are not suitable for a virtual teaching format. Therefore, we aimed to continue and provide regional anaesthesia training in a large tertiary teaching hospital in a COVID-safe manner.

Methods We implemented training of 'Plan A' blocks'. This included dedicated theatre space, ultrasound equipment and use of HDMI technology for projection. The training was delivered in small groups in a safe and socially distanced manner. Information about the delivery of content, participant satisfaction and confidence in block performance were collected using a survey. All teaching was delivered by a Consultant and trainee with interest in regional anaesthesia. Approval was obtained form the local audit department.

Results Data was collated after a four-month cycle of teaching. Attendees ranged from core trainees (67\%) to junior registrars (33\%). 95\% participants found the content, hands on experience and presenters' knowledge to be excellent. $86 \%$ of participants felt they had better confidence in their block skills after the training. Overall satisfaction with delivery of the training was recorded as excellent in $95 \%$ of candidates.

Conclusions Continuing regional anaesthesia training in the COVID-19 pandemic has been beneficial for trainees in terms of improvement in skills and to boost their morale. This has been particularly relevant as there has been a drive to reduce aerosol generating procedures and improve safety for anaesthetists and theatre staff ${ }^{2}$.

\section{DISTAL NERVE BLOCKS FOR UPPER LIMB SURGERY: HIGH QUALITY, LOW VOLUMES}

C Lopes*, D Zuzarte, M Chedas. Centro Hospitalar de Lisboa Ocidental, Lisbon, Portugal

10.1136/rapm-2021-ESRA.208

Background and Aims Through this clinical case we aim to raise awareness of the existence of highly effective peripheral nerve blocks that allow to minimize the volume of local anesthetic, thus reducing the associated complications.

Methods A 20 year-old ASA I man was admitted for urgent reconstructive plastic surgery after left hand flexor tendon injury and right forearm extensor digitorium injury.

To offer anesthesia of the whole hand, we decided to perform a left axillary block. The right-limb wound was expected to be superficial and thus manageable under subcutaneous anesthetic infiltration.

When initiating the intervention on the right wound it was obvious that subcutaneous anesthesia was insufficient as the injury was deeper than predicted.

Faced with the fact that we had almost reached the toxic dose and that we were avoiding approaching the airway, we decided to perform an ultrasound guided distal-arm radial block, as the involved structures depended on radial sensory innervation.

Results A total amount of $3 \mathrm{ml}$ of local anesthetic was used.

The patient experienced complete anesthesia of the radial nerve territory, and surgery was successfully achieved. No complications were reported

Conclusions This approach reminds us that that, similar to the distal-arm radial block, there are numerous distal blocks that can be performed. While allowing a volume sparing technique, these blocks are both easy and safe to perform, offering high quality anesthesia and analgesia. 